\section{Cahiers de Narratologie}

Analyse et théorie narratives

8 | 1997

Création de l'espace et narration littéraire

\title{
La topographie du plaisir dans Tanzai et Néadarné de Claude Crébillon
}

\section{Carmen Gómez Ramírez}

\section{(2) OpenEdition}

1 Journals

\section{Édition électronique}

URL : http://journals.openedition.org/narratologie/11643

DOI : 10.4000/narratologie. 11643

ISSN : 1765-307X

Éditeur

LIRCES

\section{Édition imprimée}

Date de publication : 1 décembre 1997

Pagination : 323-330

ISBN : 291089746X

ISSN : 0993-8516

\section{Référence électronique}

Carmen Gómez Ramírez, "La topographie du plaisir dans Tanzai et Néadarné de Claude Crébillon », Cahiers de Narratologie [En ligne], 8 | 1997, mis en ligne le 15 décembre 2020, consulté le 25 février 2021. URL : http://journals.openedition.org/narratologie/11643; DOI : https://doi.org/10.4000/ narratologie. 11643

Ce document a été généré automatiquement le 25 février 2021.

Article L.111-1 du Code de la propriété intellectuelle. 


\title{
La topographie du plaisir dans Tanzai et Néadarné de Claude Crébillon
}

\author{
Carmen Gómez Ramírez
}

\section{I}

1 En 1734 paraît chez Lou-Chou-lu-La, à Pékin, Seul imprimeur de Sa Majesté Chinoise pour les langues étrangères, Tanzaï et Néadarné. Histoire japonaise. Sous ces noms de fantaisie, il faut lire Paris où l'on imprime ces deux volumes. Et quoique l'histoire ait probablement été conçue dans les années 1732-1733, c'est en 1734 que le roman se fait connaître ${ }^{1}$. Crébillon sous lettre de cachet datant du 7 décembre de cette année-là est écroué dès le lendemain au Donjon de Vincennes. Il en sort le 13 décembre de la main d'une fidèle amie : la Princesse de Conti ${ }^{2}$. L'emprisonnement du futur Censeur Royal ${ }^{3}$ vaut à l'ouvrage une publicité remarquable: cette japonaiserie clandestine sera désormais contrefaite à Londres et aux Pays-Bas sous le titre de L'Écumoire, Histoire Japonaise. Les éditions successives publiées en France et corrigées par Crébillon gardent le titre de Tanzaï et Néadarné, et paraissent toujours anonymement.

2 Le grand succès obtenu par cet ouvrage, ce dont témoignent les nombreuses notices de presse du moment, se doit d'une part aux fortes critiques adressées à la Bulle Unigénitus et à diverses personnalités telles le Cardinal de Rohan, le Cardinal de Fleury, et la Duchesse du Maine que l'on retrouve sous les traits de la grotesque Fée Concombre. Mais dans Tanzaï, on satirise aussi le mariage de Louis XV, et le mariage tout court que Crébillon transforme en singerie. A cela s'ajoute le contenu licencieux de l'ouvrage qui raconte l'initiation douloureuse d'un jeune prince, Tanzaï, et d'une jeune princesse, Néadarné, dont l'éducation sexuelle et conjugale se fait au prix d'une double infidélité : le premier entre les griffes d'une chouette libertine, et la seconde dans les bras galants du génie Jonquille. 
Notre problématique est la suivante: découvrir comment Crébillon procède par métonymie en utilisant la main, la bouche et l'œil, et analyser comment l'enchaînement de plusieurs micro-unités narratives aboutit à la reconstruction de l'espace du corps. Nous nous attacherons donc à expliquer la procédure spatiale contenue dans Tanzaï et Néadarné qui mobilise trois niveaux de sens : les micro-unités sémantiques de base, la projection de l'image du corps et l'établissement d'une géographie de l'éros. Cette topographie du plaisir combine notamment le plaisir de toucher, le plaisir de voir et le plaisir d'aimer. En un mot, l'érographie crébillonienne s'attache comme dans la plupart de ses œuvres à combiner le plaisir de rêver d'aimer et le plaisir de conter.

\section{III}

\section{LA MAIN}

4 Il est dit que l'espace est une construction de l'homme. Pourtant le temps qui apparemment nous est donné, est fort souvent aussi une invention de l'esprit : ce que démontrèrent les séducteurs du XVIII ${ }^{e}$ en faisant du moment et de l'occasion, le temps de l'amour et du corps désiré. De cette instance physique et psychique, nous nous approcherons d'abord à partir de la main, en suivant le traitement métonymique que Crébillon lui applique.

Alors que la logique libertine s'établit dans une procédure d'extériorité de la chair, la main renvoie directement à la réalité du Corps. Ne serait-ce que par l'adhésion au Corps de la peau touchée. La main conduit le désir sur la surface épidermique, qui est par excellence la surface du réel, là où se déploient les mirages et les apparences. La main appréhende le Corps dans l'espace intériorisé de la peau, seule limite au Cosmos et à toute altérité. Ainsi après avoir réalisé un périple obligé sur le sopha, puis sur le corps de Néadarné, Jonquille obtient une réponse favorable. Et voici le commentaire qu'en fait le narrateur :

(....) Néadarné serra la main de Jonquille qui tenait la sienne, et le génie avançant à diverses reprises celle qu'il avait d'abord posée sur la jambe de la princesse, en fit usage assez indiscrètement pour qu'elle s'en fût offensé, si elle n'avait été plongée en cet instant dans la plus profonde rêverie. ${ }^{4}$

La main conduit inexorablement vers l'autre mais surtout vers le plaisir. La défaillance érotique - comme dans le cas de la princesse - est souvent au rendez-vous de l'itinérance d'une main qui accuse le vertige du désir et appartient de plein droit à la sémiologie du plaisir crébillonien. Signalons par ailleurs que le code social de l'époque confère à la main une dimension galante, conforme aux usages de la bienséance, parfaitement connus des personnages qui savent interpréter à volonté chaque geste de la main. Aussi sait-on que serrer la main est un geste indifférent dans le fond sur lequel on peut toujours s'excuser quand il ne réussit pas, mais on sait aussi que toute main qui serre veut un baiser. ${ }^{5}$

7 La main errante permet d'enrayer les distances et de transformer les parcours en voies de jouissance. La main errante devient la main galante convertie à son tour en 
démiurge topographique qui multiplie les espaces sur la base de deux d'opérations essentielles :

- le toucher, qui est la traduction du plaisir immédiat et qui amorce un mouvement allant inlassablement de la main à la bouche. ${ }^{6}$

- et le topos du chemin sillonnant le texte et les corps par lequel la main errante et galante devient la geôlière des espaces qu'elle révèle à qui veut la suivre comme Concombre, la chouette libertine, prenant le jeune Tanzaï par la main, qu'elle mène dans l'appartement des délices. ${ }^{7}$

8 Une première constatation s'impose : la main relève du concept dynamique de la distance et se matérialise dans la recherche des Corps. L'image du chemin insère la main dans la rêverie du mouvement qui privilégie le voyage voluptueux voué invariablement à l'espace du Plaisirs. La main devient le mentor plaisant de la recherche inlassable de l'expérience jouissante des sens et de l'esprit. Un tel commerce ne pouvant s'exécuter que sur la chair, le Corps devient l'espace préféré dont nous retiendrons la traverse déjà citée qui va de la main à la bouche, et que Jonquille parcours à plusieurs reprises, en faisant usage d'une main d'abord posée sur la jambe et ensuite d'une bouche charmante qu'il baisa avec ardeur. ${ }^{9}$

Le cheminement épidermique se double toujours de mots galants. Il est habituel de faire précéder l'acte d'amour par l'acte de discours. Il en résulte une triple progression inscrite sur la chair : à savoir, le parcours sensuel, le parcours discursif et le parcours écritura ${ }^{10}$. La conjugaison des différents langages qui glissent sur la surface du Corps font de la main l'un des axes de l'action libertine. Les douceurs de la main et la tendresse du discours s'expriment dans la panoplie galante de la caresse, du soupir et du baiser.

Les différents langages tactiles et sonores éclairent l'émergence progressive du Corps créé sous les mains expertes qui sculptent la silhouette désirée. Car au bout de la main c'est toujours le corps que l'on retrouve, c'est-à-dire le réel qui finit par s'imposer à la figure métonymique. Au terme de la séduction, le terme réel s'impose dans sa totalité, au détriment de la représentation par la partie. Ainsi par la main on séduit le corps, par la partie on prend possession de la totalité ce que Jonquille confirmera a Néadarné en lui disant que sa main qui n'est rien pour elle est tout pour lui ${ }^{11}$.

\section{IV}

\section{LA BOUCHE}

11 La bouche constitue la seconde unité métonymique qui nous intéresse. Elle se caractérise par une récurrence que justifient trois aspects :

- D'une part, la bouche renvoie à l'image de l'engouement et de l'avalement de l'écumoire ; c'est une allusion claire au conflit de la Bulle Unigenitus. Au niveau anecdotique, l'histoire initiale se noue à partir de cet instrument incrusté sur le corps de Tanzaï et plus tard devant être léché, c'est-à-dire avalé, par Saugrenutio le grand prêtre.

- D'autre part, la bouche représente la référence topique au sexe.

- Finalement, la bouche fonde le lieu de la Parole et de la Voix car le libertin est par les mots. Dans Tanzaï, on note par ailleurs une présence sonore constante, soit par des références à la musique, soit par le pouvoir évocateur de la voix ${ }^{12}$. 


\section{V \\ L'CEIL}

12 Nous aborderons maintenant la dernière unité, qui concerne l'œil libertin et la rêverie de la vision qui s'y rattache. Crébillon privilégie dans l'histoire de Tanzaï, la perception visuelle, ce qu'il fait aussi dans Le Sylphe, et bien sûr dans Les Egarements - pour ne citer que les ouvrages de sa toute première époque. Ce privilège du regard retient d'autant plus notre attention qu'il cible toujours le Corps, transformé en pièce centrale du spectacle galant. La mise en scène des jeux d'introspection et d'extrospection visuelles focalisent constamment l'espace épidermique.

Il ressort de ce télescopage un regard inaugural et causal - rappelons qu'il s'agit d'une histoire d'initiation - qui gère une géométrie visuelle formée à partir de la composition, de la décomposition et de la recomposition des Corps tour à tour perdus, châtiés ou transformés qui peuplent notre aventure japonaise. La création d'une telle architecture charnelle est l'aboutissement de l'interaction du regard et de l'espace, des yeux et du désir, du corps et de ses plaisirs. ${ }^{13}$

14 Le regard ne se borne pas à créer ou à recréer des espaces, les yeux s'inscrivent dans le registre complémentaire de la médiation du libertin qui sait interpréter, et dire avec les yeux qui peignaient si bien les désirs ${ }^{14}$. Le Corps désiré imprime une vision généreuse, spéculaire et médiatrice, dans l'œil libertin qui exerce sur la réalité une fascination troublante imposant à la surface lisse et univoque du réel le relief des images et des songes.

L'espace érotique est d'ailleurs inséparable chez Crébillon de la volupté de l'imagination. Rappelons à ce sujet l'ironie de Néadarné qui alla s'imaginer que Jonquille ressemblait à Tanzaï; et en s'étonnant fort (..) que cette ressemblance ne l'eut pas frappé plus tôt, elle se livra à son erreur, et par amour pour le prince, ne laissa rien à désirer à l'ardeur du génie.(..) $)^{15}$

16 Par le regard du désir, l'imagination s'impose à la volonté du logos et accélère le processus de la séduction. La scène galante développe la réplique du séducteur et de la séductrice ressourcée dans la confusion de l'imagination et de la raison appliquée au Corps. Ce corps qui est l'ouvrage du séducteur Jonquille et l'aboutissement du déploiement métonymique de Crébillon se fonde sur la fascination des yeux qui constituent le défi initiatique de tous ces personnages. Et parmi eux, c'est Jonquille qui devient le grand maître de la vision ${ }^{16}$. Ce qu'il ne manque pas de rappeler à Néadarné dans les termes suivants :

Si vous connaissiez bien ma puissance, vous ne douteriez pas que malgré tous vos refus, je ne pusse vous voir quand je le voudrais, et obtenir même de votre tendresse toutes les faveurs que vous réservez à Tanzaï. Maître de prendre sa figure, $c$ 'est sous ses traits que vous me verrez; et vous ne saurez jamais si c'est à lui, ou à moi que vous livrerez votre cœur... ${ }^{17}$

Le piège de la vision - car c'est de cela dont il s'agit-procède de la fiction appliquée au Corps. La substance visuelle est manipulée constamment par le libertin dont la volonté de jouissance ne réside pas uniquement dans le plaisir de la possession physique, mais notamment dans la possession du regard de l'Autre. Ainsi la sinuosité spatiale et la structure en écho qui caractérisent l'histoire japonaise de Crébillon attrapent le corps désiré dans le regard protéique du plaisir où l'image est le piège de toutes les volontés. 


\section{VI}

\section{CONCLUSION}

18 Si la main établit des espaces réglés et sûrs, le regard est un piège qui s'exprime par la capacité créatrice du libertin doté de mille corps car doué de raison et de pouvoir sur l'imagination. Son pouvoir s'appuie aussi sur sa maîtrise de la forme qui se traduit dans

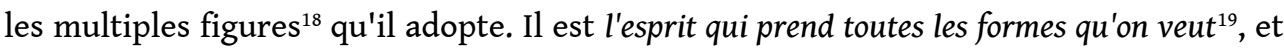
comme tel il agit sur la matière au point de laisser croire au mirage, à savoir qu'un grand ouvrage peut être celui d'un songe $e^{20}$. Beau prétexte de Jonquille pour séduire la petite Néadarné.

Le complexe processus métonymique que l'on retrouve dans Tanzaï et Néadarné développe essentiellement le topos du masque galant, le mythe de la métamorphose et l'image du corps inséparable de l'espace du songe. La topographie du plaisir s'illustre une fois de plus chez Crébillon dans le dilemme du rêve et du réel, et dans l'extrême jouissance de conter.

\section{NOTES}

1. Vid. Le catalogue des œuvres de Crébillon par Jean Sgard dans Revue d'Histoire Littéraire de la France, Janvier-février 1996, n 1, p. 3-20.

2. Vid. la chronologie de Crébillon dans Hans-Günter Funke, Crébillon fils als moralist und Gesellschaftskritiker, Heidelberg, 1972, p. 304-311.

3. Voir entre autres, Frédéric Lolié, Censure et Censeurs d'Ancien Régime. M. de Crébillon fils, dans Revue Bleue, 4e série, n 16, 1901, p. 762-766.

4. Les références bibliographiques renvoient à l'édition établie par Ernest Sturm parue chez Nizet, à Paris, en 1976. Le fragment cité renvoie à la page 128.

5. Ibidem, p. 130.

6. Ibid., p. 131 et p. 132.

7. Ibid., p. 133.

8. Ibid., p. 134.

9. Ibid., p. 135.

10. Ibid., p. 136.

11. Ibid., p. 139.

12. Ibid., p. 140.

13. Ibid., p. 167.

14. Ibid., p. 169.

15. Ibid., p. 171.

16. Ibid., p. 176.

17. Ibid., p. 177.

18. Ibid., p. 183.

19. Ibid., p. 186.

20. Ibid., p. 187. 


\section{AUTEUR}

\section{CARMEN GÓMEZ RAMÍREZ}

Université de Séville 